\title{
Neonatal Apgar Score Outcomes in Spinal Versus General Anaesthesia at the Uni- versity Teaching Hospital (UTH), Lusaka
}

\author{
Muloshi C. M.
}

\author{
Levy Mwanawasa General Hospital, Department of Anaesthesia, P.O. Box 37084, Lusaka, Zambia \\ Corresponding Author: \\ Dr Carol Musonda Muloshi: Levy Mwanawasa General Hospital, Department of Anaesthesia, \\ P.O. Box 37084, Lusaka, Zambia. Telephone: +260-977-626724: \\ Email: musondalyna@yahoo.co.uk
}

\begin{abstract}
Background: The knowledge gap was from the fact that the type of anaesthetic drug administered during caesarean section can have effects on a neonatal outcome like low Apgar score with most hospitals in developed countries now using more modern inhalational anaesthetic agents with fewer or no cardio-respiratory depressant effects, e.g. Isoflurane or Sevoflurane. However, at UTH, halothane is still being used for maintenance of general anaesthesia despite well documented cardio-respiratory depressant effects with very limited research done on its effects on the neonatal Apgar score. The Apgar score is a means of rapid evaluation of the physical condition of infants shortly after birth. Thus this study investigated the effects of general anaesthesia with halothane versus spinal anaesthesia on the neonatal Apgar score.
\end{abstract}

Objective and study design: With the view of determining the type of anaesthesia administered during caesarean section with the least effects on neonatal wellbeing shortly after birth, a prospective observational cohort study was conducted at the University Teaching Hospital between May 2015 and January 2016. The scientific objective was to determine the effects of general anaesthesia with halothane versus spinal anaesthesia on the neonatal Apgar score. This study involved 70 neonates, 54 were born from mothers that had undergone caesarean section under spinal anaesthesia and 16 from mothers that had undergone general anaesthesia.

The data was analysed using SPSS version 16.0. Inferential analysis was conducted using logistic regression.

Results: 20 neonates out of $54(37 \%)$ in the spinal anaesthetic group had an Apgar score of less than 8 at 1 minute $^{[\mathrm{Fig} 1]}$. Out of the 16 neonates in the general anaesthesia group, $14(87.5 \%)$ had an
Apgar score of less than 8 at 1 minute ${ }^{[\mathrm{Fig} 1]}$. One $(1.9 \%)$ neonate had an Apgar score of less than 8 at 5 minutes in the spinal anaesthesia group. While 4 (25\%) neonates had Apgar score less than 8 in the G.A. $\operatorname{group}^{[\mathrm{Fig} 2]}$

The logistic regression at 1 minute Showed that it is over 11 times more likely to get a low Apgar with a general anaesthetic as compared to a spinal anaesthetic (OR 11.9), [Table 3].

The Apgar score at 1 minute in the Spinal anaesthesia group was statistically significantly higher than the general anaesthesia group $(p=0.002)$. The logistic regression at 5 minutes Showed that it is over 17 times more likely to get a low Apgar with a general anaesthetic as compared to a spinal anaesthetic (OR 17.7), [Table 4]. The 5 minutes Apgar score in the spinal anaesthesia group was statistically significantly higher than the general anaesthesia group $(p=0.014)$, ${ }^{\text {Table } 4]}$.

Conclusion: With the results pointing to the fact that there is a significant difference in neonatal Apgar score outcomes in spinal versus general anaesthesia, the researcher, therefore, rejected the null hypothesis. Therefore, it is inferred that the spinal anaesthesia method has better neonatal outcomes by Apgar score measure than general anaesthesia method.

Keywords: Apgar score, Halothane, General anaesthesia and Spinal anaesthesia

\section{BACKGROUND}

\section{Introduction and literature review}

Caesarean delivery cases have been steadily increasing in recent times. The national caesarean section rate in Zambia is not documented, but the University Teaching Hospital rate was $20.6 \%$ in $2007^{[1]}$ and $25 \%$ in 2016. Choice of regional or general anaesthesia for caesarean delivery depends among others on the expertise of the 
anesthesiologist, the past medical history of the patient, choice of the patient, indication and urgency of the caesarean section ${ }^{[2]}$. Regional anaesthesia is safer for the mother and has been highly advocated for because of difficulties in airway management in pregnant women leading to difficult intubation and thus increasing maternal mortality ${ }^{[3]}$.

General anaesthesia can be carried out using different anaesthetic drugs. Most of the drugs affect the baby in two ways: direct effect from placental drug transfer and indirect effect resulting from maternal physiological and biochemical changes, which appear to be much more important. They may produce systemic effects in the baby like sedation, resulting in a low Apgar score. Present anaesthetic techniques limit the dose of intravenous agents such that fetal depression is usually not clinically significant. Drugs used for spinal anaesthesia cause a sympathetic blockage, resulting in vasodilatation and hypotension, which can reduce maternal foetal circulation and compromise the baby and cause low Apgar scores but this hypotension treated well may reduce effects on the neonate ${ }^{[4]}$.

In 1952, Dr Apgar an obstetric anaesthesiologist proposed the Apgar score as a means of rapid evaluation of the physical condition of infants shortly after the birth. When properly applied, it is a tool for standardised assessment. It also provides a mechanism to record foetal-to-neonatal transition. The scores are taken at 1 and 5 minutes after delivery. Of the two scores, the 5 minutes score is regarded as the better predictor of survival in infancy in the long term. However, the 1-minute score definitely has the value for assessing the effects of different drugs given to the mother during the Caesarean section. This method is even more appealing because it is non-invasive ${ }^{[4]}$.

Both the mother and the neonate's outcome should be considered when choosing regional or general anaesthesia for caesarean delivery. The case fatality rate for Caesarean delivery was higher in the case of general anaesthesia than regional anaesthesia ${ }^{[5]}$.

Most hospitals in high-income countries are now using more modern inhalational anaesthetic agents with fewer or no cardio-depressant effects, e.g. Isoflurane or Sevoflurane. However, at UTH, halothane is still widely used to maintain general anaesthesia and in much higher percentages despite well-documented cardio depressant effects with minimal research regarding its effects on neonatal Apgar scores ${ }^{[4]}$.

The type of anaesthetic administered during caesarean section can have effects on neonatal outcome. At UTH, halothane is still being used for maintenance of general anaesthesia during caesarean sections despite its well documented cardio-respiratory depressant effects. There has been limited research on neonatal Apgar score outcomes with general anaesthesia using halothane. In the study of Zagorzycki ${ }^{[6]}$ and $\mathrm{Aftab}^{[7]}$, a lower percentage of halothane was used because nitric oxide was also used to maintain anaesthesia. However, at UTH there is no nitrous oxide, and thus levels of halothane used to maintain anaesthesia are higher, i.e. 1 to $2 \%$ which increases the cardio depressant effects thus this study aims to bridge that knowledge gap, and the findings will help to give guidance to policymakers concerning the type and mode of anaesthetic agents which are safest for the mother and baby in our setting.

\section{MATERIALS AND METHODS}

This was a prospective observational cohort study conducted at the University Teaching Hospital in Lusaka, Zambia. The study site was the department of obstetrics and gynaecology for eight months.

The American Society of Anaesthesiologists (ASA) physical status classification is a system for assessing the fitness of patients before surgery. It has six categories with ASAI being normal healthy patients and ASAII being patients with a mild systemic illness, e.g. controlled hypertension. The target population were neonates born to ASA I or ASA II mothers undergoing urgent or elective caesarean deliveries with no prior fetal compromise admitted to UTH at or greater than 37 weeks gestation. Emergency caesarean sections were excluded from the study.

Data were collected by the use of a structured questionnaire administered by the principal researcher with the help of research assistants who were trained on how to collect the data: Maternal age, gestational age, type of anaesthesia administered (spinal or general anaesthesia), and Apgar score (at 1 minute and 5 minutes), whether neonate went to the ward with mother or to neonatal intensive care were recorded. 
The sample size was calculated using the power command in Stata version 13 with parameters derived from a study by Reynolds and Seed ${ }^{[17]:}$

At $80 \%$ power, two-sided T-test and 5\% significance level with 8:1 ratio of spinal versus general anaesthesia, a total sample size of 54 was obtained with 48 in the spinal anaesthetic group and 6 in the general anaesthetic group.

However, the duration of the study was extended by four (4) months in order to capture more patients for the general anaesthesia group, which were less common in occurrence and thus the sample size was 70, i.e. 54 in the spinal group and 16 in the general anaesthetic group.

The data was analysed using SPSS version 16.0. Inferential analysis was conducted using logistic regression.

The dependent variable was the Apgar score, and the independent variables were spinal anaesthesia and general anaesthesia.

Ethical clearance was gotten from ERES CONVERGE.

\section{RESULTS}

A total of 70 neonates were included in the study (54 in the spinal anaesthetic group and 16 in the general anaesthetic group) 20 neonates out of $54(37 \%)$ in the spinal anaesthetic group had an Apgar score less than 8 at 1 minute $^{[\mathrm{Fig} 1]}$. Out of the 16 neonates in the general anaesthesia group, $14(87.5 \%)$ had an Apgar score of less than 8 at 1 minute (Fig 1). One (1.9\%) neonate had an Apgar score of less than 8 at 5 minutes in the spinal anaesthesia group. While $4(25 \%)$ neonates had Apgar score less than 8 in the G.A. group ${ }^{[\mathrm{Fig} 1]}$.

The logistic regression at 1 minute Showed that it is over 11 times more likely to get a low Apgar with a general anaesthetic as compared to a spinal anaesthetic (OR 11.9), [Table 3].

The Apgar score at 1 minute in the Spinal anaesthesia group was statistically significantly higher than the general anaesthesia group $(p=0.002)$. The logistic regression at 5 minutes Showed that it is over 17 times more likely to get a low Apgar with a general anaesthetic as compared to a spinal anaesthetic (OR 17.7), [Table 4]. The 5 minutes Apgar score in the spinal anaesthesia group was statistically significantly higher than the general anaesthesia group $(p=0.014),{ }^{\text {Table } 4]}$.

\section{DISCUSSION}

In the spinal anaesthetic group, at 1 minute, $37 \%$ of the neonates had Apgar scores less than $8^{[\mathrm{Fig} 1]}$. For the 5 minute Apgar score, in the G.A. group, $25 \%$ of neonates had an Apgar score of less than eight compared to $1.9 \%$ neonates with an Apgar score less than 8 in the S.A. group, ${ }^{[\mathrm{Fig} 2]}$.

The 5 minute Apgar score is regarded as a better predictor of survival in infancy in the long term. Thus S.A. has lesser effects on the neonatal Apgar score at 5 minutes.

In this study, G.A. resulted in a higher number of neonates with Apgar score less than 8 at 1 minute $^{[\mathrm{Fig} 1]}$ and 5 minutes $^{[\mathrm{Fig} 2]}$, i.e. $87,5 \%$ and $25 \%$ respectively compared to S.A. which had 1 minute ${ }^{[\mathrm{Fig} 1]}$ Apgar scores of less than 8 of $37 \%$ at 5 minutes $^{[\mathrm{Fig} 2]}$ and $1.9 \%$.

The logistic regression at 1 minute Showed that it is over 11 times more likely to get a low Apgar with a general anaesthetic as compared to a spinal anaesthetic (OR 11.9), [Table 3].

The Apgar score at 1 minute in the Spinal anaesthesia group was statistically significantly higher than the general anaesthesia group $(p=0.002)$. The logistic regression at 5 minutes Shows that it is over 17 times more likely to get a low Apgar with a general anaesthetic as compared to a spinal anaesthetic (OR 17.7), [Table 4]. The 5 minutes Apgar score in the spinal anaesthesia group was statistically significantly higher than the general anaesthesia group $(p=0.014)$, $\left.{ }^{\text {Table }} 4\right]$.

This finding is different from the study that compared G.A. anaesthetic with halothane versus epidural anaesthesia on neonatal Apgar score outcome by Zagorzycki ${ }^{[6]}$ in which it was concluded that there was no difference in terms of mean Apgar score at 1 minute and 5 minutes in both the G.A. and S.A. groups.

The difference in the research outcomes of this study and the study carried out by Zagorzycki ${ }^{[6]}$ in the G.A. group can be attributed to a higher percentage of halothane, $1-2 \%$ was used in the maintenance of anaesthesia in this study despite its well-documented cardio depressant effects.

Controlling for the other variables in the multiple logistic regressions at 5 minutes $^{[\text {Table 5] }}$ for this research was considered inappropriate and attempted to demonstrate results thereof were only for information's sake to highlight the limitation of this study. The main problem was that of very small numbers of patients in the category groupings, 
i.e. low APGAR group at 5 minutes, five patients with Spinal Anaesthesia and low APGAR; only four with a General Anaesthesia and low APGAR score. This was considered from the general understanding that you need at least ten patients in each group per factor to attempt the multiple regressions. Therefore, no conclusions could be inferred from this regard at 5 minutes.

\section{Conclusion}

With the results pointing to the fact that there is a significant difference in neonatal Apgar score outcomes in spinal versus general anaesthesia, the researcher, therefore, rejected the null hypothesis. Therefore, it is inferred that the spinal anaesthesia method has better neonatal outcomes by Apgar score measure than general anaesthesia method.

\section{Acknowledgements}

My sincere gratitude goes to my supervisors Prof $\mathbf{J}$ Kinnear and Prof MPS Ngoma. Also to Dr D Bould and $\mathrm{Mr}$ M Sakala for helping with data analysis.

\section{REFERENCES}

1. Mukeshimana JM. Post caesarean section complications at the University Teaching Hospital, Lusaka. 2007.

2. Beilin Y. Anaesthesia for caesarean delivery: regional or general? In: Fleisher LA, editor. Evidence-based practice of anaesthesiology. Philadelphia: Saunders, 2004. P.401-5.

3. Bloom, SL, Spong, CY.et al (2005). Complications of anaesthesia for caesarean delivery: Obstet Gynecol, Volume 106, 2005, pp.281-287.

4. Solangi SA, Siddiqui SM, Khaskheli MS, Siddiqui MA. Comparison of the effects of general versus spinal anaesthesia on neonatal outcome. Anaesthesia Pain \& Intensive Care 2012; 16(1):18-23.

5. Dresner M. Anaesthesia for elective caesarean section. In: Pollard BJ, editor. Handbook of clinical anaesthesia. 2nd ed. Edinburgh: Churchill Livingston, 2004. p.466-9.5

6. Zagorzycki MT, Brinkman, CR. (1982). The effect of general and epidural anaesthesia upon neonatal Apgar scores in repeated caesarean section; Surg Gynecol Obstet. 1982 Nov; 155(5):641-5.
7. Aftab I, Sultan M, Masroorudin, Noor H, H.A, Khalid I.(2009). Effect of Spinal and General Anaesthesia Over APGAR Score in Neonates Born After Elective Caesarean Section.JLUMHS 9(3):151-154.

8. ACC/AHA guidelines for the management of patients with valvular heart disease. A report of the American College of Cardiology/ American Heart Association. Task Force on Practice Guidelines (Committee on Management of Patients with Valvular Heart Disease). J Am CollCardiol. 1998 Nov. 32(5):1486-588.

9. Apgar V, James L. (1962). Further observation of the newborn scoring system. American Journal of Diseases of Children. 1962; 104:419-428.

10. Baraka A, Louis F, Dalleh, R. (1990). Maternal awareness and neonatal outcome after ketamine induction of anaesthesia for Caesarean section.Canadian Journal of Anaesthesia, 37(6), 641-644.

11. Cyna AM, Dodd J. Clinical update: obstetric anaesthesia. Lancet 2007; 370:640-42.

12. Gundumure G, (2002).Characteristics and determinants of caesarean section and cord prolapsed at the University Teaching Hospital, Lusaka.

13. Idowu OA, Mafiana CF, Sotiloye D. Anaemia in pregnancy: A survey of pregnant women in Abeokuta, Nigeria. Afr Health Sci. 2005; 5:295-9.

14. Kavak ZN, Basgul A, Ceyhan N. Short term outcome of new-born infants: Spinal versus general anaesthesia for elective caesarean section: a prospective randomised study. Eur J Obstet Gynecol Rep Biol 2001; 100:50-4.

15. Ong BY, Cohen MM, Palahniuk RJ. (1989). Anaesthesia for caesarean section-effects on neonates.AnesthAnalg 1989; 68:270-5.

16. Rashid I, Behzad S, Khalid A, Tassaduq K, Imran UH, Ahmed A.Effect of general anaesthesia versus spinal anaesthesia on Apgar score in elective caesarean section, Karachi, 2012. 
17. Reynolds F, and Seed P T. (2005).Anaesthesia for Caesarean section and neonatal acid-base status: a meta-analysis. Anaesthesia, 60: 636653. doi:10.1111/j.1365-2044.2005.04223.x.

18. Rogers J F, Graves W L. (1993). Risk factors associated with low Apgar scores in a low-income population; Paediatric and perinatal epidemiology 7 (2): 205-16.

19. Tumukunde J, Lomangisi, DD, Davidson O, Kintu A, Joseph E, \&Kwizera A. (2015). Effects of propofol versus thiopental on Apgar scores in newborns and peri-operative outcomes of women undergoing emergency caesarean section: a randomised clinical trial. BMC Anesthesiology, 15, 63. http://doi. org/10.1186/s12871-015-0044-6.
21. Sahana KS, Comparison of Apgar score in neonates: spinal versus general anaesthesia for elective caesarean sections: journal of evolution of medical and dental sciences 2014: vol 3, issue 03, January 20: Page 538-543.

22. Pedersen JE, Fernandes A, Christensen M.Halothane $2 \%$ for caesarean section. Eur J Anaesthesiol.1992 Jul: 9(4):319-24.

23. Cullinane M, Gray AJG, Hargreaves, Lucas S,Max S, Herry K.M. et al. (2004). Scoping our practice, The 2004 Report of the National Confidential Enquiry into Patient Outcome and Death, NCEPOD.London. 


\section{TABLES AND FIGURES FOR THE STUDY DATA ANALYSIS}

Table 1: Ratio of general anaesthesia versus spinal anaesthesia

\begin{tabular}{|l|c|c|}
\hline Type of Anaesthesia \\
\hline General Anaesthesia & Frequency & Per cent (\%) \\
\hline Spinal Anaesthesia & 16 & 22.9 \\
\hline Total & 54 & 77.1 \\
\hline
\end{tabular}

Table 2: Type of Anaesthesia - Indication for caesarean section Cross tabulation

\begin{tabular}{|l|l|c|c|c|}
\hline \multicolumn{2}{|c|}{ Urgent } & \multicolumn{2}{c|}{ Indication for caesarean section } & \multirow{2}{*}{ Total } \\
\cline { 3 - 5 } & \multicolumn{2}{c|}{ Elective } & $16(100.0 \%)$ \\
\hline \multirow{2}{*}{ Type of Anaesthesia } & GA & $13(81.2 \%)$ & $3(18.8 \%)$ & $54(100.0 \%)$ \\
\cline { 2 - 5 } & SA & $39(72.2 \%)$ & $15(27.8 \%)$ & $70(100 \%)$ \\
\hline Total & & $52(74.3 \%)$ & $18(25.7 \%)$ & \\
\hline
\end{tabular}

Table 3: Logistic regression at 1 minute

\begin{tabular}{|l|c|c|c|c|l|l|}
\hline & & & & & \multicolumn{2}{|c|}{ 95\% C.I. for OR } \\
\cline { 5 - 7 } & B & Std. Error & p-value & OR & Lower & Upper \\
\hline Anaesthesia & 2.477 & 0.807 & 0.002 & 11.900 & 2.448 & 57.843 \\
\hline
\end{tabular}

Note: $\mathrm{OR}=$ Odds ratio

Table 4: Logistic Regression at 5 minutes

\begin{tabular}{|l|l|l|l|l|l|l|}
\hline & & & & \multicolumn{2}{|c|}{ 95\% C.I. for OR } \\
\cline { 5 - 7 } & B & Std. Error & p-value & OR & Lower & Upper \\
\hline Anaesthesia & 2.872 & 1.163 & 0.014 & 17.667 & 1.809 & 172.571 \\
\hline
\end{tabular}

Note: $\mathrm{OR}=\mathrm{Odd}$ ratio

Table 5: Multiple Logistic Regression at 1 minute

\begin{tabular}{|l|c|c|c|c|c|c|c|}
\hline & \multirow{2}{*}{$\mathrm{B}$} & \multirow{2}{*}{ St.Error } & \multirow{2}{*}{ Wald } & \multirow{2}{*}{ p-value } & \multirow{2}{*}{ Exp(B) } & \multicolumn{2}{|c|}{ 95.0\% C.I.for EXP(B) } \\
\cline { 7 - 9 } & & & & & & Lower & Upper \\
\hline Type of Anaesthesia & 2.872 & 0.875 & 10.777 & 0.001 & 17.666 & 3.181 & 98.108 \\
\hline Time (Incision to Delivery) & -0.126 & 0.107 & 1.390 & 0.238 & 0.881 & 0.714 & 1.087 \\
\hline Gestation Age & 0.127 & 0.141 & 0.804 & 0.370 & 1.135 & 0.861 & 1.496 \\
\hline Age of Mother & 0.064 & 0.062 & 1.042 & 0.307 & 1.066 & 0.943 & 1.204 \\
\hline Indication for surgery & 0.010 & 0.631 & 0.000 & 0.987 & 1.010 & 0.293 & 3.483 \\
\hline Parity & -0.103 & 0.249 & 0.172 & 0.679 & 0.902 & 0.553 & 1.470 \\
\hline Constant & -10.978 & 6.212 & 3.123 & 0.077 & 0.000 & & \\
\hline
\end{tabular}




\section{Apgar Score at 1 minute versus Type of Anaesthesia}

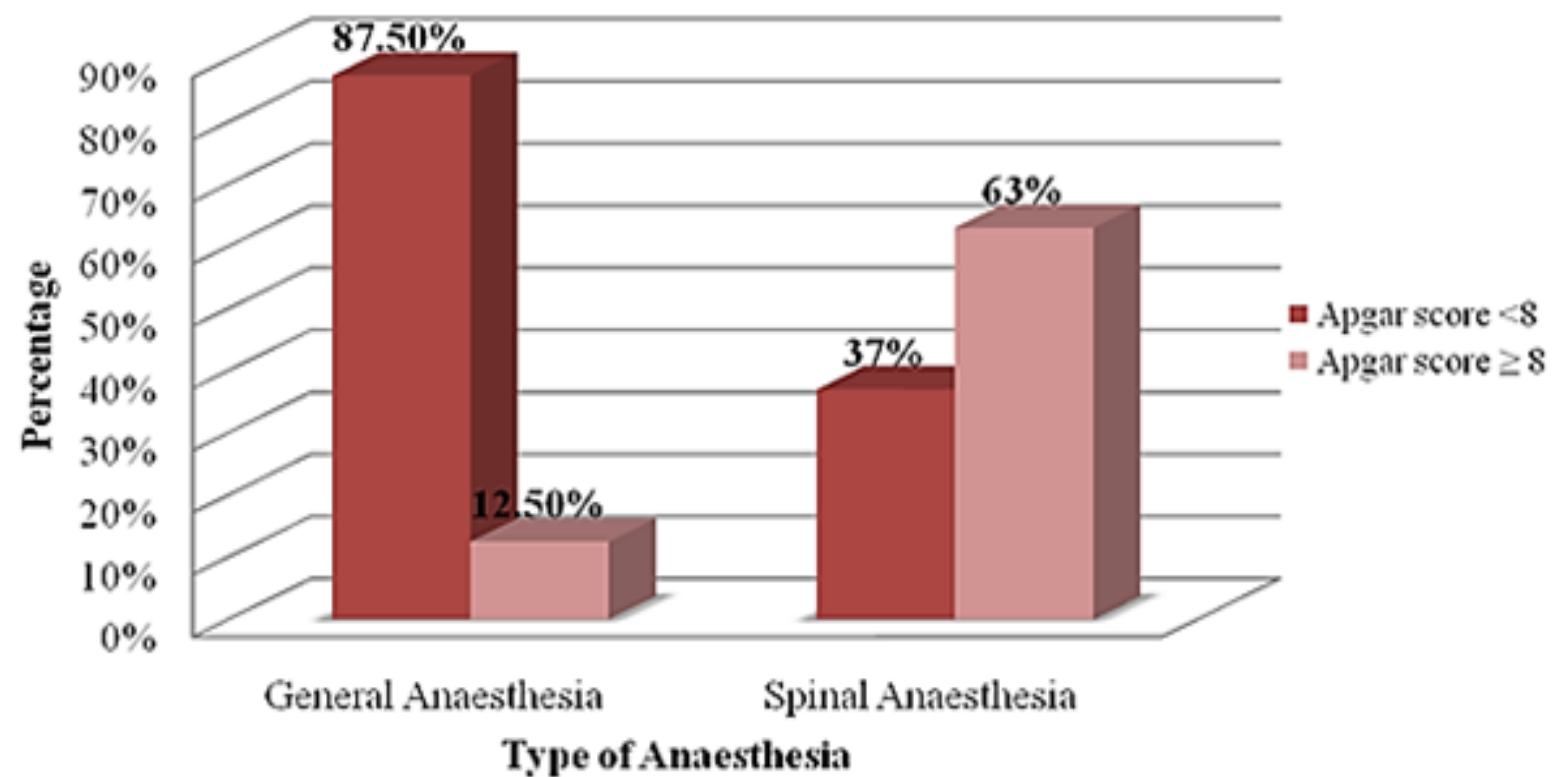

Figure 1: Apgar score at 1 minute versus type of anaesthesia

\section{Apgar Score at 5 minutes verses Type of Anaesthesia}

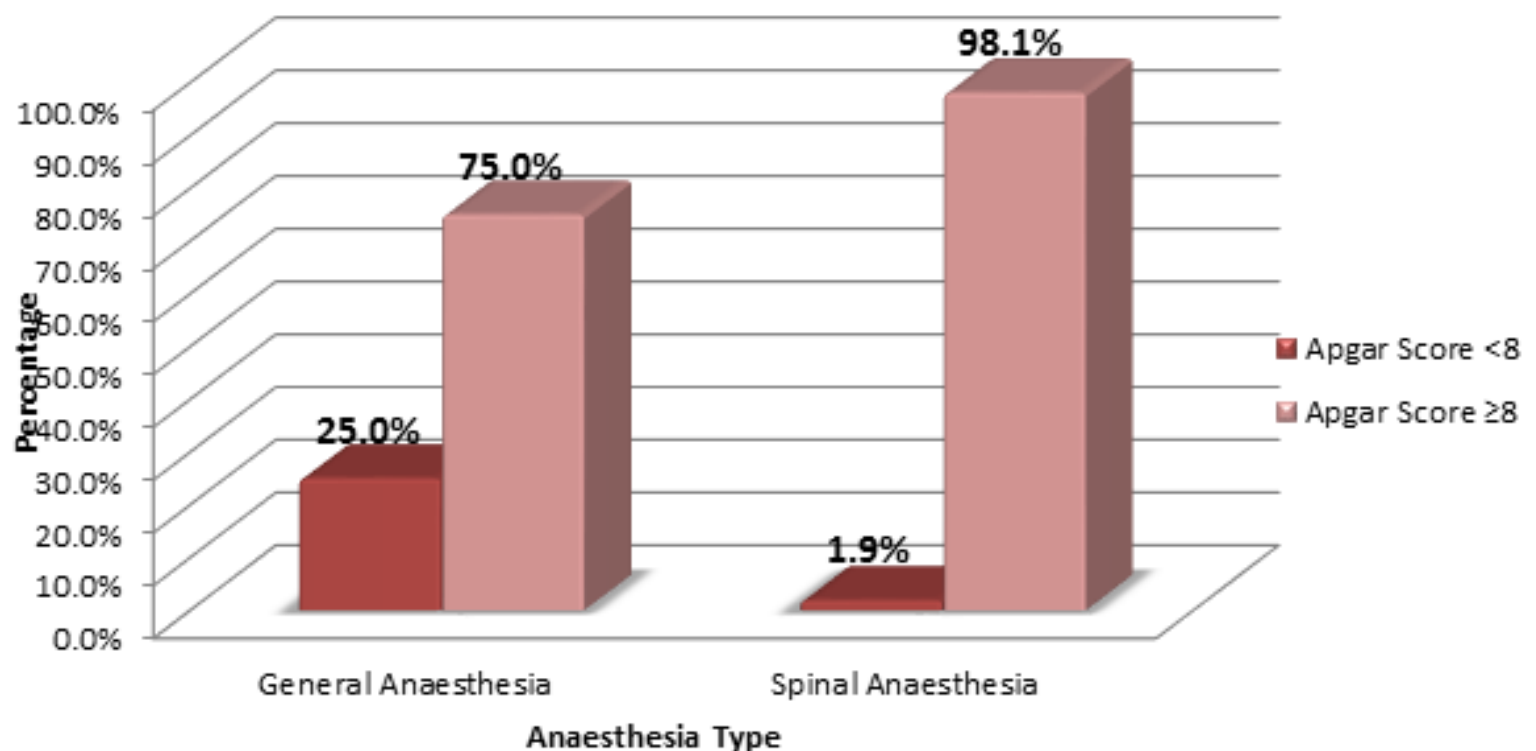

Figure 2: Apgar score at 5 minutes versus the type of anaesthesia 